\section{Tumor Necrosis Factor Inhibitors Provide Longterm Clinical Benefits in Pediatric and Young Adult Patients with Blau Syndrome}

To the Editor:

Blau syndrome is an autoinflammatory disease caused by mutations in the NOD2 gene ${ }^{1}$. The initial symptoms of Blau syndrome usually include polyarthritis and rash followed by uveitis. In addition, various clinical manifestations beyond the classic clinical triad have been reported in patients with Blau syndrome $e^{2,3,4}$.

Although controlling ocular and articular involvements are critical to improve prognosis, no specific therapy for Blau syndrome has yet been established. The use of thalidomide, immunosuppressives [methotrexate (MTX) or azathioprine], and biologic therapy [tumor necrosis factor- $\alpha$ (TNF- $\alpha$ ) or interleukin (IL)-1 $\beta$ inhibitor] has been reported in corticosteroid-refractory cases ${ }^{1,4,5,6}$; however, the small number of patients with Blau syndrome in these studies and case reports makes drawing a definite conclusion difficult.

To address these issues, we report a series of 6 patients ( 3 men and 3 women) with Blau syndrome who received biologic therapy between 2005 and 2013 at the Kagoshima University Hospital, Kagoshima, Japan. Clinical findings and laboratory data were collected from their medical records. No patient had achieved clinical remission with prior treatments, including nonsteroidal antiinflammatory drugs, MTX, and systemic corticosteroids. In all cases, the patients or their legal guardians provided written informed consent before administration of biologic therapy. The publication of the patients' data was approved by the Institutional Review Board at Kagoshima University Hospital (No. 26-129).

Patients' baseline characteristics are shown in Table 1. Median age at the start of biologic therapy was 14.0 years (5.3-20.8). Median age at disease onset and median disease duration were 1.7 years $(0.5-4.5)$ and 13.4 years
(0.8-18.1), respectively. Four of 6 patients received oral corticosteroids (1-15 mg/day) in combination with MTX. Articular manifestations were observed in all 6 patients, and the most commonly affected joints were the wrist, followed by the proximal interphalangeal and metacarpophalangeal joints. Three patients had active uveitis and 2 of them also had panuveitis, whereas 2 younger patients (cases 4 and 5) were uveitis-free at baseline. Lower extremity erythema nodosum was observed in 3 of 6 patients.

Clinical course and outcome are summarized in Table 2. Four patients showed a favorable response to TNF- $\alpha$ inhibitors: infliximab (IFX; cases 1 and 2), adalimumab (ADA; case 3), or etanercept (ETN; case 4) used as the first biologic agent. In contrast, 1 patient (case 5) discontinued ETN followed by tocilizumab (TCZ) owing to a lack of clinical efficacy. Another patient (case 6) developed anti-TCZ immunoglobulin E (IgE) antibodies and discontinued TCZ. Both cases 5 and 6 achieved clinical remission after switching to IFX. Changes in inflammatory biomarkers such as erythrocyte sedimentation rate, $\mathrm{C}$-reactive protein, and matrix metalloproteinase 3 between baseline and the last visit are shown in Appendix 1.

In our case series, all patients had achieved clinical remission by the last visit. Overall, TNF- $\alpha$ inhibitors showed a favorable effect, except for ETN in 1 patient.

TNF- $\alpha$ inhibition with IFX or ADA has shown successful results in a few patients with Blau syndrome $e^{7,8}$, which is in agreement with our findings. In contrast, IFX, ADA, or ETN were inefficacious in several patients ${ }^{1}$; further, ETN-induced myelopathy has been reported in a pediatric patient with Blau syndrome ${ }^{9}$. Use of the IL-6 receptor inhibitor TCZ has not been reported in patients with Blau syndrome. In our study, TCZ was administered to 2 patients, but was discontinued because of recurrent arthritis or development of anti-TCZ IgE antibodies. This might have resulted from the absence of co-therapy with MTX, which is used in combination with other biologic drugs; nevertheless, its efficacy remains unclear. Taken together, biologic therapy might be a promising approach, but is not always effective

Table 1. Characteristics of patients at initiation of treatment with biologic agents.

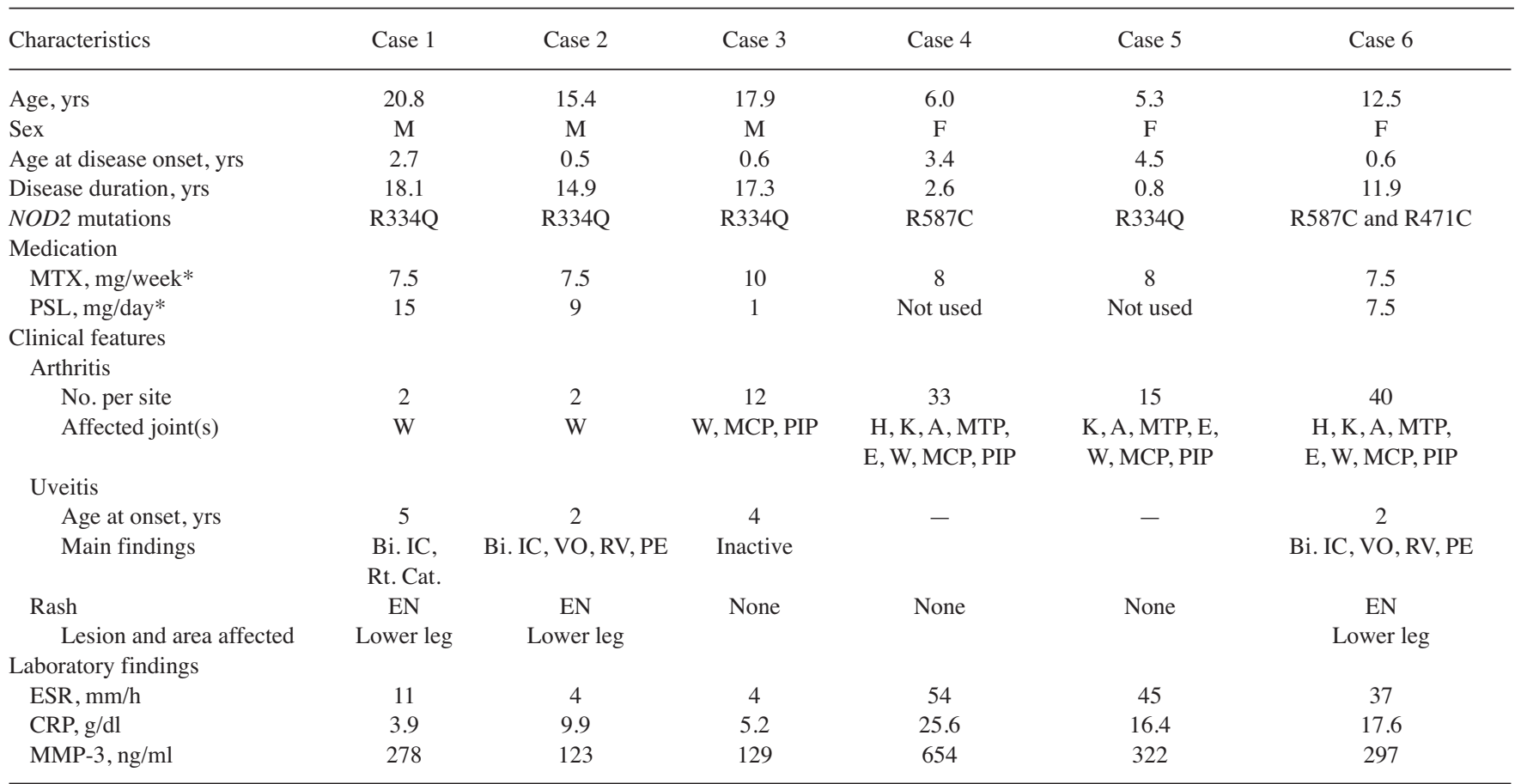

* MTX or PSL was given orally; cases 4 and 5 had never received systemic corticosteroids previously. MTX: methotrexate; PSL: prednisolone; ESR: erythrocyte sedimentation rate; CRP: C-reactive protein; MMP-3: matrix metalloproteinase 3; W: wrist; MCP: metacarpophalangeal; PIP: proximal interphalangeal; H: hip; K: knee; A: ankle; MTP: metatarsophalangeal; E: elbow; Bi.: bilateral; IC: iridocyclitis; Rt.: right; Cat.: cataract; VO: vitreous opacity; RV: retinal vasculitis; PE: papilledema; EN: erythema nodosum. 
Table 2. Clinical course and outcome with biologic therapy.

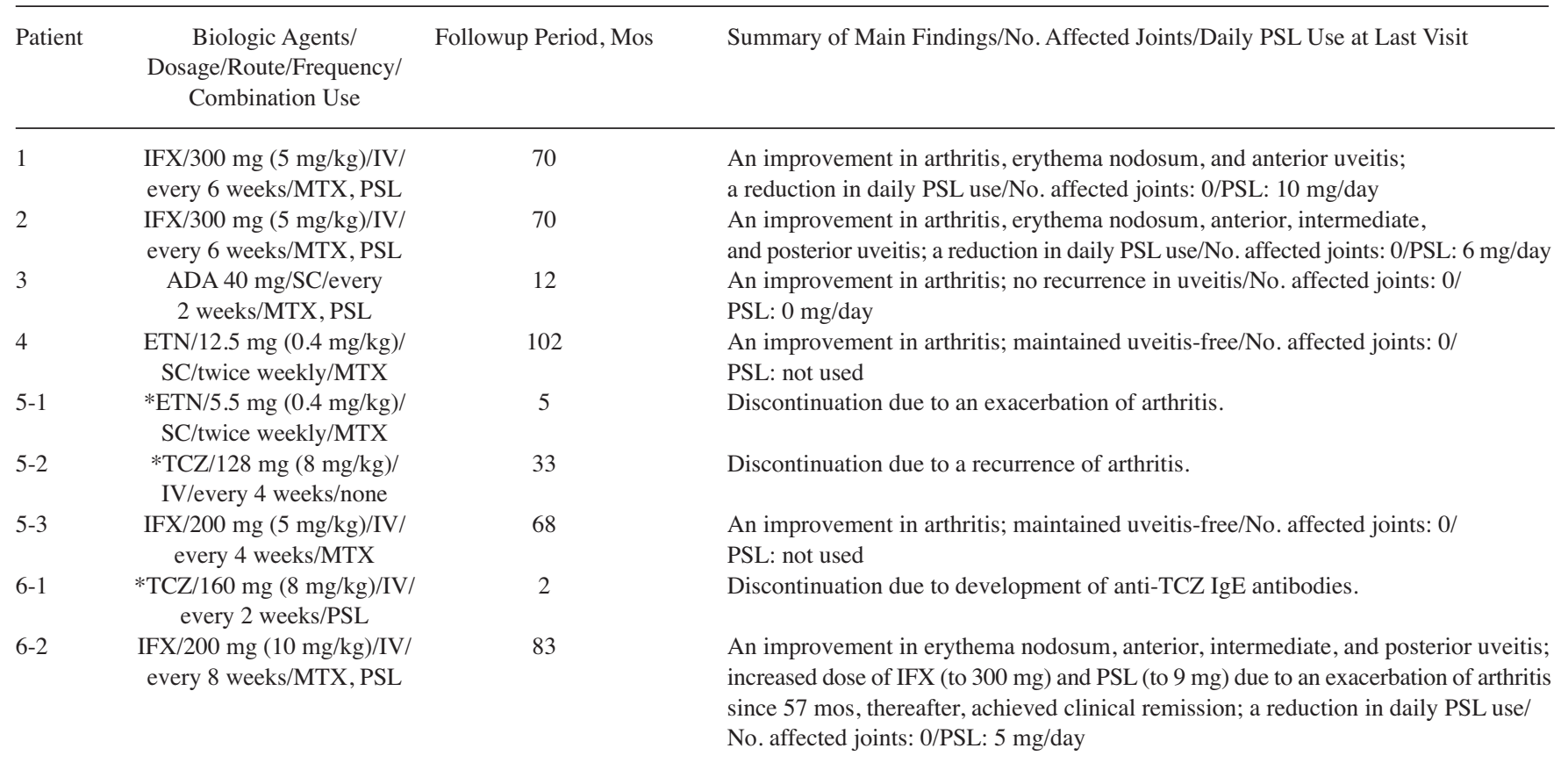

\footnotetext{
* Discontinued and switched to the second or third biologic agent. MTX was given orally once a week; cases 4 and 5 were treated without corticosteroids. IFX: infliximab; IV: intravenous; MTX: methotrexate; PSL: prednisolone; ADA: adalimumab; SC: subcutaneous injection; ETN: etanercept; TCZ: tocilizumab; IgE: immunoglobulin E.
}

in patients with Blau syndrome. Moreover, we should pay careful attention to the adverse effects of biologic agents.

An international multicenter study by Rosé, et $\mathrm{l}^{4}$ including 18 children and 13 adults, with a median age of 16.5 years (range 1.9-58) showed that despite administration of systemic steroids and immunosuppressive and/or biologic drugs (ADA or IFX), about $60 \%-70 \%$ of the patients still had active arthritis and severe ocular involvement at baseline. Thus, Blau syndrome is resistant to currently available therapies in many cases.

Early intervention with biologic therapy may be a possible reason for the positive results we obtained because our study enrolled pediatric and young adult patients. Indeed, 2 younger patients (cases 4 and 5) who started biologic therapy at the ages of 6 years and 5 years, respectively, were maintained uveitis-free without the administration of systemic corticosteroids. Otsubo, et al reported the cases of a Japanese mother and her daughter with Blau syndrome who had contrasting outcomes; the daughter obtained clinical remission with TNF- $\alpha$ inhibitor while her mother missed the opportunity for biologic therapy because of far-advanced disease ${ }^{10}$. These results suggest that early intervention is essential to improve patients' quality of life and disease prognosis.

TNF- $\alpha$ inhibitor may be a promising approach in the management of Blau syndrome. However, our present study has limitations, including the retrospective design and the small sample size.

\section{ACKNOWLEDGMENT}

The authors thank their colleagues at the Kagoshima University Hospital for their valuable advice and encouragement. The authors also acknowledge the help received from the Department of Pediatrics, Kyoto University Graduate School of Medicine, with the NOD2 gene analysis. We thank Editage for English-language editing.

TOMOKAZU NAGAKURA, MD, The Holy Mother of Grace Hospital Home for Children and Persons with Severe Motor and Intellectual Disabilities, Usuki; HIROYUKI WAKIGUCHI, MD, PhD, Department of Pediatrics, Kagoshima University Graduate School of Medical and Dental
Sciences, Kagoshima, and Department of Pediatrics, Yamaguchi University Graduate School of Medicine, Ube; TOMOHIRO KUBOTA, MD, Department of Pediatrics, Kagoshima University Graduate School of Medical and Dental Sciences, Kagoshima; TSUYOSHI YAMATOU, MD, Department of Pediatrics, Kagoshima University Graduate School of Medical and Dental Sciences, Kagoshima; YUICHI YAMASAKI, MD, $\mathrm{PhD}$, Department of Pediatrics, Kagoshima University Graduate School of Medical and Dental Sciences, Kagoshima; YUKIKO NONAKA, MD, Department of Pediatrics, Kagoshima University Graduate School of Medical and Dental Sciences, Kagoshima; YASUHITO NEROME, MD, Department of Pediatrics, Kagoshima University Graduate School of Medical and Dental Sciences, Kagoshima; HARUMI AKAIKE, MD, Department of Pediatrics, Kagoshima University Graduate School of Medical and Dental Sciences, Kagoshima; TOMOKO TAKEZAKI, MD, Department of Pediatrics, Kagoshima University Graduate School of Medical and Dental Sciences, Kagoshima; HIROYUKI IMANAKA, MD, Department of Pediatrics, Kagoshima University Graduate School of Medical and Dental Sciences, Kagoshima; YOSHIFUMI KAWANO, MD, $\mathrm{PhD}$, Department of Pediatrics, Kagoshima University Graduate School of Medical and Dental Sciences, Kagoshima; SYUJI TAKEI, MD, Department of Pediatrics, Kagoshima University Graduate School of Medical and Dental Sciences, Kagoshima, and School of Health Sciences, Faculty of Medicine, Kagoshima University, Kagoshima, Japan. This work was supported by a Health Labor Sciences Research Grant from the Ministry of Health, Labor and Welfare of Japan. Address correspondence to Dr. H. Wakiguchi, Department of Pediatrics, Yamaguchi University Graduate School of Medicine, 1-1-1 Minamikogushi, Ube, Yamaguchi 755-8505, Japan. E-mail: hiroyuki@yamaguchi-u.ac.jp

\section{REFERENCES}

1. Caso F, Costa L, Rigante D, Vitale A, Cimaz R, Lucherini OM, et al. Caveats and truths in genetic, clinical, autoimmune and autoinflammatory issues in Blau syndrome and early onset sarcoidosis. Autoimmun Rev 2014;13:1220-9. 
2. Rosé CD, Wouters CH, Meiorin S, Doyle TM, Davey MP, Rosenbaum JT, et al. Pediatric granulomatous arthritis: an international registry. Arthritis Rheum 2006;54:3337-44.

3. Rosé CD, Aróstegui JI, Martin TM, Espada G, Scalzi L, Yagüe J, et al. NOD2-associated pediatric granulomatous arthritis, an expanding phenotype: study of an international registry and a national cohort in Spain. Arthritis Rheum 2009;60:1797-803.

4. Rosé CD, Pans S, Casteels I, Anton J, Bader-Meunier B, Brissaud P, et al. Blau syndrome: cross-sectional data from a multicentre study of clinical, radiological and functional outcomes. Rheumatology 2015;54:1008-16.

5. Aróstegui JI, Arnal C, Merino R, Modesto C, Antonia Carballo M, Moreno $\mathrm{P}$, et al. NOD2 gene-associated pediatric granulomatous arthritis: clinical diversity, novel and recurrent mutations, and evidence of clinical improvement with interleukin-1 blockade in a Spanish cohort. Arthritis Rheum 2007;56:3805-13.

6. Martin TM, Zhang Z, Kurz P, Rosé CD, Chen H, Lu H, et al. The NOD2 defect in Blau syndrome does not result in excess interleukin-1 activity. Arthritis Rheum 2009;60:611-8.
7. La Torre F, Lapadula G, Cantarini L, Lucherini OM, Iannone F. Early-onset sarcoidosis caused by a rare CARD15/NOD2 de novo mutation and responsive to infliximab: a case report with long-term follow-up and review of the literature. Clin Rheumatol 2015;34:391-5.

8. Chauhan K, Michet C. A case of Blau syndrome. Case Rep Rheumatol 2014;2014:216056.

9. Caracseghi F, Izquierdo-Blasco J, Sanchez-Montanez A, Melendo-Perez S, Roig-Quilis M, Modesto C. Etanercept-induced myelopathy in a pediatric case of blau syndrome. Case Rep Rheumatol 2011;2011:134106.

10. Otsubo Y, Okafuji I, Shimizu T, Nonaka F, Ikeda K, Eguchi K. A long-term follow-up of Japanese mother and her daughter with Blau syndrome: Effective treatment of anti-TNF inhibitors and useful diagnostic tool of joint ultrasound examination. Mod Rheumato 2017;27:169-73

J Rheumatol 2017;44:4; doi:10.3899/jrheum.160672

APPENDIX 1. Changes in patients' laboratory variables. Laboratory variables at baseline and at last visit were compared. Overall, inflammatory biomarkers decreased in all patients. However, ESR and MMP-3 levels remained above normal range at the last visit (ESR for patients 4 to 6; MMP-3 for patients 1,5, and 6). ESR: erythrocyte sedimentation rate; MMP-3: matrix metalloproteinase 3; CRP: C-reactive protein.

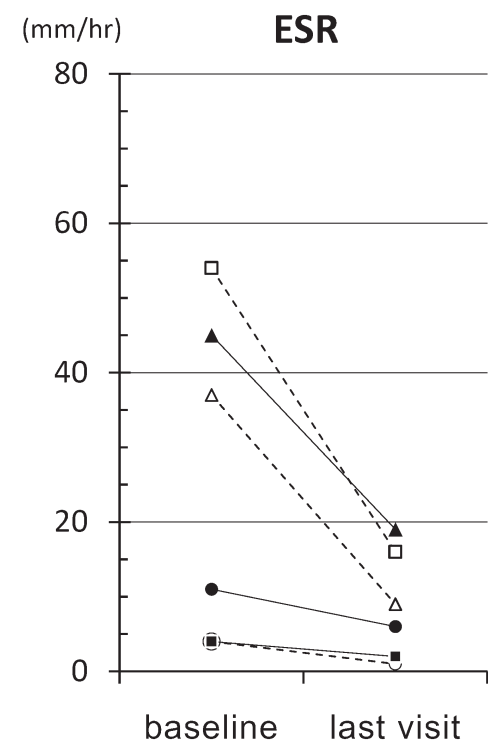

$(\mathrm{g} / \mathrm{dL})$

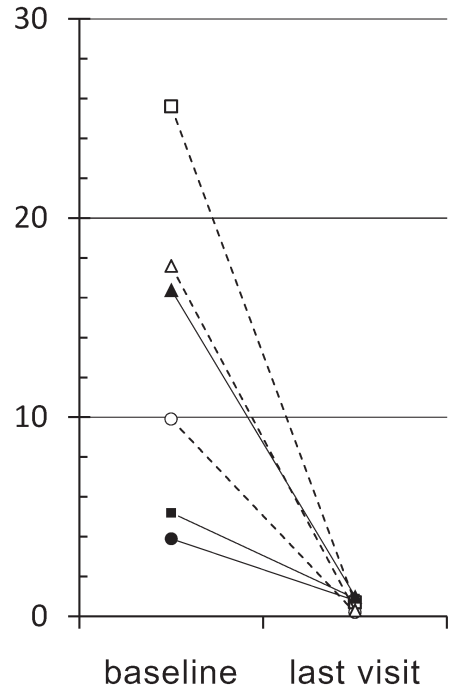

MMP-3

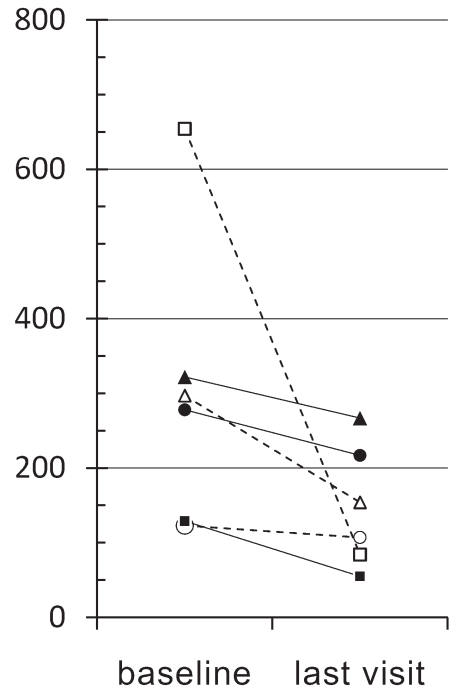

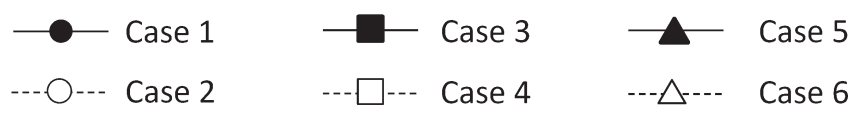

